\title{
Covid-19: Doctors in Norway told to assess severely frail patients for vaccination
}

\author{
Ingrid Torjesen
}

Doctors in Norway have been advised to assess severely frail and terminally ill patients to determine whether the benefits of vaccination outweigh the risks of possible side effects, after reports indicated that vaccine side effects may have led to deterioration and death of some patients.

Reports of 33 suspected adverse drug reactions with fatal outcomes after administration of the Pfizer and BioNTech vaccine had been received by the Norwegian Medicines Agency as at 17 January. All the people who died were over 75. Around 42000 people are believed to have received the first dose of the vaccine so far in Norway.

The agency has investigated 13 of the deaths so far and concluded that common adverse reactions of mRNA vaccines, such as fever, nausea, and diarrhoea, may have contributed to fatal outcomes in some of the frail patients. ${ }^{1}$

Although for most elderly frail people any side effects of the vaccine will be outweighed by a reduced risk of a severe covid-19 disease, for those with the severest frailty even relatively mild side effects can have serious consequences, the Norwegian Institute of Public Health said. And the benefits of the vaccine to those with a very short life expectancy may be marginal or irrelevant, it said. The institute is now recommending that doctors carefully consider the benefits and disadvantages of giving the vaccine to extremely frail patients (such as those whose frailty is ranked 8 or 9 on the Clinical Frailty Scale or equivalent) and terminally ill patients ahead of vaccination.

Azeem Majeed, professor of primary care and public health at Imperial College London, said, “The experience of Norway shows the importance of monitoring the safety and efficacy of the new covid-19 vaccine. They are now being given to millions of people globally, including many people who will be older and frailer than the participants in the clinical trial.

"Some older people would be expected to die, and what we need to know is how the observed death rate in older vaccine participants compares with the expected death rate (taking into account factors such as frailty and morbidity). Without this information, it's difficult to draw any conclusions and give older people evidence based information on the safety of the vaccines."

The UK was the first country to roll out the Pfizer-BioNTech vaccine, but the Medicines and Healthcare Products Regulatory Agency has yet to publish any data on adverse reactions to it.
The World Health Organization said that it had been in touch with the Norwegian authorities and the European Medicines Agency to get more information on the deaths that occurred. "As soon as WHO and partners have gained a full understanding of these events, the findings and any changes to current recommendations will be immediately communicated to the public," it said.

Covid-19 vaccines have been given emergency licences and are being rolled out quickly to protect people at high risk of serious illness and to achieve herd immunity, said Raj Bhopal, emeritus professor of public health at the University of Edinburgh, adding, "These principles are absolutely correct, but we mustn't forget other vital principles, including autonomy and informed consent.”

He added, "What is happening in Norway is probably happening in other countries. The MHRA will surely be taking the Norwegian reports seriously and examining the data here. Side effects and adverse effects are common but have not been presented to the public in detail that helps to get everyone on board quickly. But that's a short term strategy, and open and transparent communication is required in the longer term."

Adverse effects such as headaches, fatigue, muscle pains, and fever are common with covid-19 vaccines, and it was important to be clear about that, Bhopal said, but he knew of people who had been shown the data sheet listing potential adverse effects only after they had received the -19 vaccine, one of whom told him, "If I had had the chance to read that data sheet before I was vaccinated I would have had some questions."

Bhopal added, "In due course, if it turns out people have adverse effects, and then they come back and say, 'But I didn't give informed consent-I wasn't told about these adverse effects,' that could, unfortunately, undermine the vaccination programme.”

In a statement Pfizer UK said, "We are working with the Norwegian Medicines Agency (NOMA) to gather all the relevant information.

"Norwegian authorities have prioritised the immunisation of residents in nursing homes, most of whom are very elderly with underlying medical conditions and some who are terminally ill. NOMA confirm the number of reports so far is not alarming and in line with expectations. All reported deaths will be thoroughly evaluated by NOMA to determine if these reports are related to the vaccine. Norwegian health authorities have now changed their recommendation in relation to vaccination of the terminally ill (Clinical Frailty Scale 8 or higher). 
"Our immediate thoughts are with the bereaved families.”

Severest end of Clinical Frailty Scale ${ }^{2}$

- 8 Very severely frail: Completely dependent, approaching the end of life. Typically, they could not recover even from a minor illness.

- 9 Terminally ill: Approaching the end of life. This category applies to people with a life expectancy less than six months, who are not otherwise evidently frail.

1 Torjesen I. Covid-19: Norway investigates 23 deaths in frail elderly patients after vaccination. BMJ2021;372:n149. doi: 10.1136/bmj.n149 pmid: 33451975

2 NICE. Clinical Frailty Scale. https://www.nice.org.uk/guidance/ng159/resources/clinical-frailtyscale-pdf-8712262765.

This article is made freely available for use in accordance with BMJ's website terms and conditions for the duration of the covid-19 pandemic or until otherwise determined by BMJ. You may use, download and print the article for any lawful, non-commercial purpose (including text and data mining) provided that all copyright notices and trade marks are retained. 